\title{
Contextual issues that influence prepared
nurses for critical care nursing practice in Malawi
}

\author{
Rodwell Gundo ${ }^{1}$, Gael Mearns ${ }^{1}$, Annette Dickinson ${ }^{1}$, \\ Ellen Chirwa ${ }^{2}$
}

1. Auckland University of Technology, Auckland, New Zealand

2. University of Malawi, Kamuzu College of Nursing, Lilongwe, Malawi

\section{Background}

Abstract

There are no critical care nurse training programs in Malawi despite the high burden of diseases which culminate in critical illness. This paper presents contextual issues that influence preparedness of nurses for critical care nursing practice in Malawi. The qualitative findings presented are part of a larger mixed methods study which explored learning needs of critical care nurses as a way of informing the development of a training program for the critical care nurses in Malawi.

Methods

Interpretive descriptive design was used. Data were gathered through 10 key informant interviews with nurse leaders ( $\mathrm{n}=8$ ) and anaesthetists $(\mathrm{n}=2)$; and two focus group discussions with registered nurses and nurse midwife technicians working in intensive care and adult high dependency units at two tertiary hospitals. Transcribed data were analyzed manually and through the use of NVivo data management software utilizing Thorne's steps of analysis ${ }^{1}$.

Results

Being unprepared to work in intensive care and high dependency units was a dominant theme. Factors that contributed to this sense of unpreparedness were lack of educational preparation, organisational factors and workforce issues. The consequences of nurses' perceptions of being unprepared were fearfulness, a change of nurses' attitudes and elevation of risk to patients. The nurses managed unpreparedness by relying on other health professionals and learning on the job.

\section{Conclusion}

The findings illuminated contextual issues to be considered when developing programs for upskilling nurses in hospitals within Malawi and contributes to the developing body of knowledge related to nursing education and practice development within developing countries.

Key words: Critical Care Nursing; Critical Illness; Developing Countries; Malawi; Education, Nursing; Qualitative Research; Critical Care

\section{Introduction}

Critical care practice in developing countries is still in its infancy. Despite the high burden of illness and the likelihood that this will increase ${ }^{2}$ there are numerous challenges that negatively affect the care of critically ill patients in the developing countries. The identified challenges include lack of prehospital care, delayed access to critical care, inadequate infrastructure for critical care and lack of physicians and nurses with critical care training ${ }^{3,4,5}$. The lack of competent staff including nurses is considered one of the biggest challenges $^{6}$. The lack of competent staff could possibly be attributed to the lack of training programs specific for critical care nurses in developing countries like Malawi ${ }^{7}$.

In the absence of well-trained professionals like physicians and anaesthetists, nurses perform extended roles such as intubating patients ${ }^{8,9}$. However, undergraduate nursing training alone is not sufficient for nurses to perform these extended roles $^{10}$. Because of the lack of post-registration critical care training programs, nurses rely on intuition and basic nursing education to identify and care for the critically ill patients ${ }^{9,11}$. Prior studies conducted in Malawi reported that nurses in intensive care unit (ICU) and high dependency unit (HDU) lack knowledge and skills (i.e. competence) in certain aspects of intensive and critical care nursing practice ${ }^{11,12,13}$. For example, a study by Mula, Ncama and Maluwa ${ }^{11}$ on enteral feeding reported that majority of the nurses failed to undertake precautions such as checking gastric residual volume and daily inspection of nostrils to prevent tube feeding complications.

This research was a qualitative study conducted as a component of a larger mixed methods study which explored learning needs of nurses in ICUs and HDUs as a way of informing the development and evaluation of a training program for nurses in Malawi. While discussing learning needs of the nurses, participants described issues which impacted critical care nursing practice in the country. These findings illuminated the contextual factors that influence critical care nursing practice in Malawi.

\section{Methods}

\section{Research design}

An interpretive descriptive design was used in the qualitative component of the explanatory sequential mixed methods design. An interpretive descriptive design aims to generate knowledge for clinical applications while also acknowledging the researcher's foreknowledge about the phenomenon understudy as a useful starting point to orient the research ${ }^{14,15}$. The impetus for this current study was documented evidence, though limited, that nurses in Malawi lack knowledge and skills (i.e. competence) required for the care of critically 
ill patients and their families in ICU/HDU ${ }^{11,12,13}$. These findings also aligned with the primary author's observations while working as a critical care nurse in Malawi.

\section{Study context}

The study was conducted in ICUs and adult HDUs at two tertiary hospitals in Malawi (Hospital A and B). At the time of data collection, there were three adult HDUs and a fivebedded general ICU at Hospital A; six adult HDUs and fourbedded ICU at Hospital B.

\section{Participants}

A purposive sampling technique was used to identify participants for the focus group discussion (FGD) and key informant interviews. FGDs participants were recruited from $79 \mathrm{ICU} / \mathrm{HDU}$ nurses who participated in an initial survey conducted as part of the larger study; 13 of the 79 ICU/HDU nurses participated in two FGD interviews. Participants for key informant interviews, nurse leaders (n $=8)$ and anaesthetists $(\mathrm{n}=2)$ were invited to contribute their perspective on nurses' learning needs because the nurse leaders supervise the nurses and anaesthetists work along the nurses in the units. See Table 1 for demographic profile of the focus group and key informant interview participants.

\section{Data collection}

The FGDs and key informant interviews were conducted in English at the most convenient time and place for the participants but within the hospitals. Malawi is an Anglophone country where English is compulsory in schools and colleges. As such, health professionals in Malawi are fluent in English after a minimum of 12 years of formal education and a minimum of 3 years of nursing or health related training. Of note, the term "nurse" for the purposes of this study and for reporting study findings refers to both registered nurses and nurse midwife technicians (NMT). NMTs are trained at the Diploma level and they constitute the largest nursing and midwifery cadre in Malawi ${ }^{16}$. They are expected to provide general patient care and conduct uncomplicated deliveries in areas where senior nurses and midwives are limited in number ${ }^{17}$. Registered nurses and NMTs constituted separate FGDs to ensure homogeneity of the groups.

Semi-structured questions guided the interviews. Participants were encouraged to elaborate on learning needs of the nurses in ICUs/HDUs and share their experiences and issues that affected their learning and practice in the units. All FGDs and interviews were transcribed verbatim by either the primary author or a registered nurse research assistant.

\section{Data analysis}

The transcribed data were entered into NVivo to aid data management. The data were also analysed manually, and the process was informed by Thorne's steps of analysis'. The initial stages of analysis involved familiarization with and immersion in the data; achieved through critical reading and reflecting on the interview transcripts. Next followed preliminary coding where the initial codes were identified and progressively refined during an iterative process of recoding and analysis. After organizing data into various groups, the next step involved making sense of relationships between the various groups. Key decisions, questions and mind maps were recorded in a notebook. The notes helped to refine ideas, groups and relations as data collection and analysis progressed. The identified codes and relationships between groups of codes were shared and discussed with supervisors to support trustworthiness of the findings.

\section{Ethical consideration}

The study was approved by Auckland University of Technology Ethics Committee in New Zealand (reference number 15/439) and National Health Sciences Research Committee in Malawi (ref NHSRC \#1533). In addition, permission was sought from the Hospital Director at each hospital and individual participants. Participants were given information letter with details of the study. The participants who expressed willingness to participate in the study were requested to give written consent. Anonymity and confidentiality were maintained by requesting the participants to use pseudonyms of their choice. In addition, the researcher emphasized the importance of not sharing details of the discussion with other people. The authors have access to the data according to ethics privacy requirements.

Table 1: Demographic profile of focus group and key informant interview participants

\begin{tabular}{lccc}
\hline Variable & Category & $\begin{array}{c}\text { Focus Group } \\
\text { Discussion } \\
\text { Participants }\end{array}$ & $\begin{array}{c}\text { Key Informant Interview } \\
\text { Participants }\end{array}$ \\
& & $(\mathrm{N}=13)$ & $(\mathrm{N}=10)$ \\
\hline Age & Mean & 30 years & 43 years \\
Experience & Mean & 2 years & 14 years \\
Gender & Male & 2 & 2 \\
Qualification & Female & 11 & 8 \\
& Master's Degree & - & 5 \\
& Degree & 6 & 4 \\
Professional & Registered Nurse/ & 6 & 1 \\
cadre & Midwife & & 8 \\
& Nurse Midwife & 7 & - \\
& Technician & & \\
Training in & Anaesthetist & - & 2 \\
Critical Care & Yes & - & 2 \\
Nursing & & & \\
& & & \\
& No & 13 & 5 \\
Facility & Not applicable & - & 5 \\
& Hospital A & 7 & 5 \\
Type of unit & Hospital B & 6 & 5 \\
& ICU & 2 & 5 \\
\hline & HDU & 11 & 5 \\
\hline
\end{tabular}

\section{Results}

Being unprepared to work in ICU and HDUs was a dominant theme within this study. This referred to the perception of nurse leaders, anaesthetists and nurses (RNs and NMTs) that the nurses did not have the required knowledge and/or skills to safely and competently care for critically ill patients. Factors that contributed to this sense of unpreparedness included a lack of educational preparation as well as organisational factors. In addition, limited resources (e.g. medications, monitors, suction catheters and perfusors) influenced the lack of preparation and was a common finding across all identified themes. The lack of preparedness caused fear, change of nurses' attitude and elevation of risk to the 
patients. The nurses managed unpreparedness by relying on other health professionals and learning on the job.

\section{Educational preparation}

The nurse leaders, anaesthetists and nurses observed that the nurses in ICUs and HDUs did not possess any post registration critical care nursing education qualification and had not attended any short courses on critical care nursing. Basic nursing education was considered inadequate to prepare the nurses for critical care practice because the ICUs and HDUs are specialised areas which provide monitoring and support to critically ill patients. This was expressed by one of the nurse leaders;

"...most of the times the nurses that are placed in the ICU, they are not trained as ICU nurses and they have not undergone even any short course or a training on what they are expected to do, the care that they are supposed to provide to critically ill patients..." (Participant 10)

Nurse leaders reported that the majority of the nurses at the two hospitals were NMTs who were trained at Diploma level. They were considered inadequately prepared to work in the highly-specialised units. The nurse participants readily admitted that they lacked the knowledge, skills and experience required for practice in the units because they were not educationally qualified in critical care nursing. The nurses further observed that some of the knowledge and skills acquired during undergraduate training were either not sufficient or different from actual practice in critical care settings. For example, the nurses reported that they learnt about tracheostomy care but they lacked the skill to perform the task. Working in the units was perceived to be challenging because nurses were expected to perform to the level of a trained critical care nurse;

"...When you are working in ICU as a nurse, every person who comes to the unit expects you to do things like a trained critical care nurse not knowing that you don't have knowledge, skills and resources. It's like you have a limit so there are high expectations from us. Since we don't have adequate appropriate knowledge and experience, it becomes very challenging" (Participant 14)

\section{Organisational factors}

Participants reported that the annual rotation system implemented by senior nurses who are responsible for manging nursing services at the two hospitals resulted in some nurses being transferred between different departments and wards every year. The nurses were deployed to any ward or unit including the ICU and HDU depending on need, regardless of the nurse's preferred career path. Nurse leader and anaesthetist participants observed that this annual rotation led to loss of nurses with experience. One anaesthetist explained;

"... the experienced ones... got used to ICU...now they are moved to the ward, for example going to paediatric ward or outpatient department, so these new nurses...for them to acquire some basic knowledge and skills, it takes time and that in the process is also compromising the care of the patient." (Participant 9)

The rotation of experienced nurses to other departments or wards left few, or in some instances, no experienced nurses who could supervise and support new nurses in the ICU and HDUs. The new nurses who had been transferred from other wards were not comfortable to work in either the ICUs or the HDUs because they considered these units as complex requiring special nursing skills which the nurses did not have. A participant explained;

"I remember the day that I was told to move to HDU, I refused and even told the Matron that maybe you should move me to the ward... I was not comfortable to work in the HDU because I could see that I didn't have the necessary skills... When I looked at the set up in HDU, I asked myself...what am I going to do here?" (Participant 13)

\section{Challenge of resources}

Participants observed that a critical shortage of nurses at the two hospitals led to deployment of newly qualified nurses in large number to specialized units like the ICU and the HDU. The new graduate nurses worked without support from experienced nurses because as previously noted they were not available in most units. As a result, the new nurses relied on anaesthetists to teach them, and while able to support them in regard to medical and technical aspects of care, the anaesthetists were not conversant with the nursing care of critically ill patients as noted by one of the nurse leaders;

"...they need to go through a lot of training or work under somebody who is experienced. At the moment we have been privileged to work with anaesthetists who most of the times are there. Here and there, they might help us, but they are not conversant with nursing component. As a result, there are a lot of issues in relation to competence. Most of the nurses are not competent and some even mention it that they are not competent." (Participant 22)

Apart from the shortage of nurses, some nurses also described that the lack of support staff, namely porters and hospital attendants negatively affected the delivery of nursing care in the units. In Malawian hospitals, porters are responsible for transferring patients between departments and deceased patients from the ward or unit to the mortuary. Hospital attendants are responsible for cleaning the unit or ward and in some cases, they also assist with the transfer of patients and deceased patients. The absence of support staff meant that the nurses, already working with heavy workloads, had to assume these roles often placing other patients at risk as recounted by a nurse from HDU;

"I think as nurses we have a lot of work to do. We tend to be porters, sometimes we tend to be maids [hospital attendants] in addition to caring for our patients. Hospital authorities should allocate permanent porters who will be responsible for transferring patients to and from different departments. Imagine, sometimes we leave the patients and the whole ward just to transfer a dead body to the mortuary..." (Participant 3)

Furthermore, the two hospitals and subsequently the units lacked material resources such as medications, monitors, suction catheters, perfusors, masks, gloves, colostomy bags and urine bags. The lack of resources was contrary to nurses' expectations. The unpreparedness of the nurses led to fearfulness, change of nurses' attitudes and an elevation of risk to the patients. The nurse leaders noted that fearfulness was common among nurses who had no previous experience and felt unprepared to work. Supporting the nurse leaders' observations, nurses admitted that they felt fearful and unprepared when they were told to transfer from a ward to 
HDU as described by this nurse;

"I was working in paediatric ward before being transferred to HDU... The time I was being transferred, I had a lot of fear. I used to ask myself, what am I going to do?' (Participant 2)

Nurse leaders observed that the attitude of some nurses towards patients and guardians* (In Malawi, the term guardian refers to a patient's family member or significant other) was bad. Some nurse leaders suspected that the bad attitude was a response to the nurses' feeling of inadequacy as one nurse leader explained,

'Because you don't know what to do, at the end of the day the patient is hurt, or the guardian is burt because they are not being talked to in a good way so the attitude as well is not good". (Participant 10)

The lack of appropriate knowledge and skills among the nurses who worked in HDUs increased the risk to the critically ill patients. Nurses admitted that they were unable to perform some nursing interventions because they did not know what to do or how to operate the equipment used in HDU. The latter is illustrated in the following example of one nurse;

"... we used to have patients discharged from ICU and the ICU staff used to tell us that, maybe this patient needs a perfusor but because we didn't know how to set the perfusor the patient could not get the medication". (Participant 7)

Despite the unwillingness to work in the units the nurses realised that they had to respect decisions made by authorities and work in these units. The nurses relied on physicians or anaesthetists for guidance on the care of critically ill patients, learning 'on the job' from visiting expatriates, colleagues, through self-directed learning and observing.

\section{Discussion}

The study explored learning needs of nurses in ICUs and HDUs to inform development of a training program for nurses in Malawi. Participants cited lack of educational preparation in critical care nursing, organisational factors, shortage of staff and lack of resources as challenges affecting critical care nursing practice in the country. In addition, the participants observed that there is discrepancy between preservice training and the actual nursing practice in ICUs and HDUs. These findings corroborate results of previously cited studies which reported that ICU and HDU nurses in Malawi lacked knowledge and some skills required for the care of critically ill patients ${ }^{11,12,13}$. Reasons are offered for these findings. There are no training programs specific for critical care nurses in Malawi. This is in contrast to middle and high-income countries where post-registration critical care nursing programs are prevalent ${ }^{7}$. At the time of data collection, there were less than 10 nurses who had been formally trained in critical care nursing outside the country. Unfortunately, some of these nurses were no longer working in the clinical areas having joined training institutions or moved into management positions at the two hospitals.

Another possible explanation for the nurses' lack of preparedness is the lack of guidelines for in-service training or continuing professional development (CPD) especially in the public sector. This leads to uncoordinated in-service trainings within and across Government, donors and nongovernmental organisations ${ }^{18}$. Nurses in Malawi are expected to earn 25 points on CPD annually as a requirement for renewal of registration with Nurses and Midwives Council of Malawi ${ }^{19}$. However, the topics for the CPD session are left to the discretion of the CPD coordinator and may not be related to critical care nursing practice. Our findings highlight the need for post registration critical care nursing programs and on-the-job training tailored to the needs of nurses in ICUs and HDUs. Further work is required to align the curriculum for basic nursing education with current critical care practice. These initiatives would help to address the deficit of specially trained critical care nurses in the country.

Furthermore, participants observed that majority of nurses in ICUs and HDUs were NMTs who were considered not well trained for practice in critical care settings. This is also in contrast to developed countries like Australia where majority of nurses who work in these units are registered nurses trained at degree level ${ }^{20}$. As earlier indicated, NMTs are the largest nursing and midwifery cadre in Malawi ${ }^{16}$. However, in most instances NMTs work outside their scope of practice, a process called task shifting because of shortage of senior nurses trained at degree or higher level. Unfortunately, their NMT training does not cover the content which is expected to be covered by higher cadres $^{16,21}$. These findings highlight the need to train nurses to a higher level to work in highly specialist areas.

Our study established that the nurses acquired some of the required knowledge and skills from their colleagues and through self-directed learning. Surprisingly, the two hospitals have a policy on annual rotation which gives nurse leaders mandate to transfer some nurses from one hospital department including ICUs and HDUs to another department. Participants of this study observed that the practice leads to loss of some nurses who had gained experience in the ICUs and HDUs. This finding is consistent with previous studies in Malawi which reported that competency gaps are aggravated by an annual rotation of nurses ${ }^{22}$. Therefore, the findings of the present study add voice to the calls for authorities to revisit the annual rotation system to ensure optimal care provision.

The reports about shortage of nurses are not surprising because Malawi is one of the developing countries experiencing critical shortage of health professionals. The vacancy rate for all health workers is reported to be $45 \%$. The vacancy rate of nursing officers (RNs with minimum of first degree in nursing) and NMT is $66 \%$ and $60 \%$ respectively. The shortage is attributed to inadequate intakes in training institutions due to diminishing government funding for preservice training ${ }^{18}$, international migration and in-country migration between public and private health sectors in search for better pay, between urban and rural areas and between tertiary and primary health care delivery $y^{23,24}$. The shortage of staff leads to high workload especially for nurses who are at the frontline of health service delivery. The findings support calls for strategies to retain nurses and hire more support staff in the health system. The lack of resources for the care of critically ill patients in developing countries has been reported in several studies ${ }^{2,25}$. The shortage of resources and technologies in Malawi is attributed to inadequate health https://dx.doi.org/10.4314/mmj.v31i2.6 
financing, weak supply chain management, irrational use of medicines and pilferage ${ }^{18}$. Although Malawi is a signatory to The Abuja Declaration which calls on African Governments to increase their budgetary allocation to health to at least $15 \%$ of the national budget, health financing is currently below the stipulated $15 \%{ }^{17}$. Health financing depends on external funding from development partners. For instance, the development partners contributed $61.6 \%$ of total health expenditure during the period 2012/13-2014/15 ${ }^{18}$. The lack of resources negatively affects the delivery of health services which include critical care ${ }^{26,27}$. While we support calls for increased health financing, further research is required to explore innovative ideas to improve patient care utilizing the available resources. In the present study, participants report that the unpreparedness of the nursing workforce in ICUs and HDUs resulted in attitudinal changes among the nurses. In addition, there was increased risk to the critically ill patients. The report of fear amongst nurses is similar to findings from other studies that note stress and increased anxiety amongst critical care nurses because of critical illness of the patients and the presence of technology which requires technical competence ${ }^{28,29,30}$. Equipment is perceived as a resource that facilitates nursing care. However, as noted in this and other studies, it becomes an obstacle to patient care when nurses are unable to operate the equipment due to lack of knowledge ${ }^{31}$. This study confirmed the findings of previous studies conducted at the same hospitals that nurses in ICU and HDUs also underestimated the complex needs of family members. The contributing factors were lack of training in critical care nursing, lack of policies and lack of preparedness to deal with the family members ${ }^{12,13}$.

\section{Conclusion}

The findings of this study show that lack of educational preparation on critical care nursing, organisational factors, shortage of staff and lack of resources negatively affect critical care nursing practice in Malawi. The transferability of these findings is subject to certain limitations. For instance, the sample was small, and the study was conducted at two public tertiary hospitals. In spite of these limitations, the findings have important implications for critical care education and practice in Malawi. The findings also extend existing knowledge on critical care practice in developing countries.

\section{Acknowledgement}

The study was conducted in fulfilment of the primary author's degree of Doctor of Philosophy at Auckland University of Technology (AUT) with funding from New Zealand Foreign Affairs and Trade Aid Program. Additional research funding was provided by AUT and National Commission for Science and Technology in Malawi. Preliminary findings were presented at the $13^{\text {th }}$ World Congress of Intensive and Critical Care Medicine in Brazil in November 2017. The authors declare that there is no conflict of interest.

\section{Author's contribution}

RG contributed to conceptualization of the overall study from which this paper has been developed, study design, data collection, data analysis, drafting and revision of the manuscript. GM, AD and EC supervised the study, provided guidance and critical feedback at every stage of the study. In addition, GM, AD and EC provided comments on the initial draft of the manuscript and contributed to the revision of the manuscript.

\section{References}

1. Thorne S. Interpretive Description. California, United States of America: Left Coast Press, Inc: 2008

2. Adhikari NKJ, Fowler RA, Bhangwanjee S, Rubenfeld GD. Critical care and the global burden of critical illness in adults. Lancet. 2010 Oct;376(9749):1339-1346. doi: 10.1016/S0140-6736(10)60446-1

3. Baker T, Lugazia E, Eriksen J, Mwafongo V, Irestedt L, Konrad D. Emergency and critical care services in Tanzania: A survey of ten hospitals. BMC Health Serv Res. 2013 Apr 16;13:140. doi:10.1186/1472-6963-13-140

4. Stafford RE, Morrison CA, Godfrey G, Mahalu W. Challenges to the provision of emergency services and critical care in resourceconstrained settings. Glob Heart. 2014 Sep;9(3):319-323. doi:10.1016/j. gheart.2014.08.005

5. Ntogwiachu D, Suiyven ED, Williams G. Critical care nursing in Cameroon: formation of the Cameroon Association of Critical Care Nurses. Connect. 2014;9(3):115-117

6. Vukoja M, Riviello E, Gavrilovic S et al. A survey on critical care resources and practices in low-and middle-income countries. Glob Heart. 2014 Sep;9(3):337-342. doi:10.1016/j.gheart.2014.08.002

7. Barnes J, Paterson-Brown L. Improving care of critically unwell patients through development of a simulation programme in Malawian Hospital. J Edu Training Studies. 2017;5(6):90-96. doi:10.11114/jets. v5i6.2366

8. Jochberger S, Ismailova F, Banda D et al. A survey of the status of education and research in anaesthesia and intensive care medicine at the University Teaching Hospital in Lusaka, Zambia. Arch Iran Med. 2010;13(1):5-12.

9. Carter C, Snell D. Nursing the critically ill surgical patient in Zambia. Br J Nurs. 2016 Nov 10;25(20):1123-1128. doi:10.12968/ bjon.2016.25.20.1123

10. Skees J. Continuing education: A bridge to excellence in critical care nursing. Crit Care Nurs Q 2010 Apr-Jun;33(2):104-116. doi:10.1097/ CNQ.0b013e3181d913a1

11. Mula C, Ncama BP, Maluwa A. Nurses' competency and challenges in enteral feeding in the Intensive Care Unit (ICU) and High Dependency Unit (HDU) of a referral hospital, Malawi. Malawi Med J. 2014 Sep;26(3):55-59.

12. Gondwe WTM, Bhengu BR, Bultemeier K. Challenges encountered by intensive care nurses in meeting patients' families' needs in Malawi. Afr J of Nurs Midwifery. 2011;13(2):92-102

13. Gundo R, Bodole F, Lengu ES, Maluwa A. Comparison of nurses' and families' perception of family needs in critical care unit at referral hospitals in Malawi. Open J Nurs. 2014;4(4):312-320. doi:10.4236/ ojn.2014.44036

14. Thorne S, Kirkham SR, O'Flynn-Magee K. The analytic challenge in interpretive description. Internatl J Qualitative Methods. 2004;3(1):111

15. Hunt MR. Strengths and challenges in the use of interpretive description: Reflections arising from a study of the moral experience of health professionals in humanitarian work. Qual Health Res. 2009 Sep;19(9):1284-1292. doi:10.1177/1049732309344612

16. Holman J. The role of nurse midwife technicians in task shifting in Malawi. Thesis submitted to University of Washington. 2012 [cited 2018 June 17]. Available from https://digital.lib.washington. edu/researchworks/bitstream/handle/1773/21960/Holman washington_0250O_10970.pdf;sequence $=1$

17. Government of Malawi. Malawi Health Sector Strategic Plan 
2011 - 2016. Lilongwe: Ministry of Health. 2011 [cited 2015 August 20]. Available from http://www.nationalplanningcycles.org/sites/ default/files/country_docs/Malawi/2_malawi_hssp_2011_-2016_final_ document_1.pdf

18. Government of Malawi. Health Sector Strategic Plan II (20172022). Lilongwe: Ministry of Health. 2017 [cited 2018 June 17]. Available from http://www.nationalplanningcycles.org/sites/default/ files/planning_cycle_repository/malawi/health_sector_strategic_plan_ ii_030417_smt_dps.pdf

19. Nurses and Midwives Council of Malawi. Continuing Professional Development. 2016 [cited 2018 July 01]. Available from http://www. nmcm.org.mw/cpd.html

20. Hegney D, Tuckett A, Parker D, Robert E. Access to and support for continuing professional education amongst Queensland nurses: 2004 and 2007. Nurse Educ Today. 2010 Feb;30:142-149. doi:10.1016/j. nedt.2009.06.015

21. Jacob S, Holan J, Msolomba R et al. Using a task analysis to strengthen nursing and midwifery pre-service education in Malawi. Int J Nurs Midwifery. 2015;7(5):84-103. doi:10.5897/IJNM2015.0132

22. Harris L, Fioratou E, Broadis E. Paediatric burns in LMICs: An evaluation of the barriers and facilitators faced by staff involved in burns education training programmes in Blantyre, Malawi. Burns. 2016 Aug;42(5):1074-1081. doi:10.1016/j.burns.2015.04.011

23. Government of Malawi. Nurse/Midwife training operational plan, field assessments, analysis and scale-up plans for nurse training institutions. Lilongwe. 2011 [cited 2015 June 03]. Available from https://www.k4health.org/sites/default/files/Malawi_National\%20 Nurse\%20Training\%20Operational\%20Plan.pdf

24. Manafa O, McAuliff E, Maseko F, Bowie C, MacLachlan M, Normand C. Retention of health workers in Malawi: Perspectives of health workers and district management. Hum Resour Health. $2009 \mathrm{Jul}$
28;7:65. doi:10.1186/1478-4491-7-65

25. Murthy S, Leligdowicz A, Adhikari NKJ. Intensive care unit capacity in low-income countries: A systematic review. PLoS One. 2015;10(1):e0116949. doi:10.1371/journal.pone.0116949

26. Manda-Taylor L, Mndolo S, Baker T. Critical care in Malawi: The ethics of beneficence and justice. Malawi Med J. 2017 Sep;29(3):268271. doi:10.4314/mmj.v29i3.8

27. Abdu M, Wilson A, Mhango C, Taki F, Coomarasamy A, Lissauer D. Resource availability for the management of maternal sepsis in Malawi, other low- income countries, and lower- middle- income countries. Int J Gynaecol Obstet. 2018 Feb;140(2):175-183. doi:10.1002/ijgo.12350

28. Gohery P, Meaney T. Nurses' role transition from the clinical ward environment to the critical care environment. Intensive Crit Care Nurs. 2013 Dec;29(6):321-328. doi:10.1016/j.iccn.2013.06.002

29. Monks J, Flynn M. Care, compassion and competence in critical care: A qualitative exploration of nurses' experience of family witnessed resuscitation. Intensive Crit Care Nurs. 2014 Dec;30(6):353-359. doi:10.1016/j.iccn.2014.04.006

30. Bagherian B, Sabzevari S, Mirzaei T, Ravary A. Meaning of caring from critical Care nurses' perspective: A Phenomenological Study. Journal Intensive \& Crit Care. 2017:3(3:33). doi:10.21767/24718505.100092

31. Tunlind A, Granstrom J, Engstrm A. Nursing care in a hightechnological environment: Experience of critical care nurses. Intensive Crit Care Nurs. 2015 Apr;31(2):116-123. doi:10.1016/j. iccn.2014.07.005 